\title{
CÂNCER DO ESÔFAGO - COMPLICAÇÕES PÓS-OPERATÓRIAS IMEDIATAS E LETALIDADE HOSPITALAR
}

\section{ESOPHAGEAL CANCER - IMEDIATE POSTOPERATIVE COMPLICATIONS AND HOSPITAL MORTALITY}

\author{
Danilo Gagliardi, ACBC-SP ${ }^{1}$ \\ Paulo Roberto Corsi, TCBC-SP ${ }^{2}$ \\ Carlos Estevão Frimm, ECBC-SP ${ }^{3}$ \\ João Fava, TCBC-SP ${ }^{3}$
}

\begin{abstract}
RESUMO: Objetivo: O objetivo deste estudo é verificar as variáveis que podem influenciar as complicações pósoperatórias imediatas e a mortalidade hospitalar dos pacientes portadores de câncer do esôfago submetidos a tratamento cirúrgico. Método: Por meio de análise retrospectiva de 60 pacientes verificamos, pela análise uni e multivariada, se variáveis como procedência, tempo de história, doenças prévias, tabagismo, etilismo, perda ponderal, localização do tumor, tipo de cirurgia realizada, estádio da doença e caráter da operação poderiam exercer alguma influência sobre as complicações e os óbitos constatados. Resultados: Verificamos que, em relação às variáveis resultantes, complicações pleuropulmonares, sepse, deiscência de anastomose cervical, mediastinite e óbito, as variáveis explanatórias mais significativas foram, respectivamente: cirurgia paliativa, mediastinite, tumor localizado no segmento torácico superior e sepse. As variáveis explanatórias estudadas não tiveram valor significativo na análise univariada em relação à resultante deiscência de anastomose torácica. Constatamos que, em relação à variável resultante, complicações pleuropulmonares, as explanatórias associadas mais significativas foram: cirurgia paliativa e síndrome da angústia respiratória do adulto (S.A.R.A.). A interdependência dessas variáveis permite afirmar que, nos portadores de câncer do esôfago submetidos à cirurgia paliativa e que desenvolveram S.A.R.A., as complicações pleuropulmonares foram 13,8 vezes mais freqüentes. Conclusão: A cirurgia paliativa e a localização do tumor no segmento superior correlacionaram-se com as complicações pleuropulmonares registradas. Nenhuma das variáveis estudadas correlacionou-se com as deiscências de anastomose intratorácica.
\end{abstract}

Descritores: Neoplasias esofágicas; Esôfago; Esofagectomia; Complicações pós-operatórias; Mortalidade hospitalar.

\section{INTRODUÇÃO}

Apesar do grande progresso nas áreas afins da cirurgia do esôfago e do esmero técnico dos cirurgiões, as complicações e a letalidade hospitalar no câncer do esôfago continuam elevadas.

Em relação às complicações pós-operatórias, independentemente do tipo de cirurgia realizada, as de natureza respiratória e as deiscências de anastomose, associadas ou não à mediastinite e/ou sepse, são as mais freqüentemente referidas na literatura como causas conseqüenciais dos óbitos. ${ }^{1-5}$

Os fatores de risco que podem influenciar a ocorrência das complicações pleuropulmonares, das deiscências de anastomose e da letalidade hospitalar, no pós-operatório da cirurgia do câncer do esôfago, são estudados aleatoriamente, tendo como base a casuística pessoal. Entre eles encontramos: idade, sexo, grau de disfagia, doenças associadas, localização e/ou extensão do tumor, tipo histológico, dosagem

1. Professor Assistente-Doutor do Departamento de Cirurgia da Faculdade de Ciências Médicas da Santa Casa de São Paulo.

2. Professor Assistente do Departamento de Cirurgia da Faculdade de Ciências Médicas da Santa Casa de São Paulo.

3. Professor Titular - Livre Docente do Departamento de Cirurgia da Faculdade de Ciências Médicas da Santa Casa de São Paulo.

Recebido em 15/03/2002

Aceito para publicação em 21/11/2003

Trabalho realizado no Departamento de Cirurgia da Faculdade de Ciências Médicas da Santa Casa de São Paulo. 
de albumina, estado nutricional e/ou deficiência imunológica, quimioterapia e/ou radioterapia, cirurgia realizada e/ou a duração desta, resposta hormonal ao trauma cirúrgico, tipo de anastomose (manual ou mecânica) e/ou número de planos de sutura, perda sangüínea operatória, caráter da cirurgia, estádio da doença, complicações pleuropulmonares e deiscências das anastomoses. ${ }^{1,4-14}$

$\mathrm{Na}$ tentativa de correlacionar a atuação associada ou independente dos fatores relatados com as complicações pleuropulmonares, deiscências das anastomoses e letalidade hospitalar, alguns autores utilizaram a análise multivariada no tratamento estatístico de seus resultados ${ }^{1,4,5,8-12}$. As conclusões obtidas foram diversas, em virtude da seleção de diferentes variáveis.

Assim sendo, o objetivo deste trabalho foi, através da análise retrospectiva de pacientes portadores de neoplasias malignas do esôfago, verificar se algumas variáveis podem influenciar as complicações pós-operatórias imediatas e a letalidade hospitalar.

\section{MÉTODO}

Estudamos 60 doentes portadores de câncer do esôfago, 58 pertencentes ao Departamento de Cirurgia da Faculdade de Ciências Médicas da Santa Casa de São Paulo e dois à clínica privada, atendidos num período de oito anos. Eram do sexo masculino 42 doentes (70\%). A idade variou de 39 a 77 anos, com média de 58,2 anos.

O diagnóstico foi realizado através de esofagograma e esofagoscopia com biópsia. Constatou-se carcinoma espinocelular em 59 casos e adenocarcinoma em um.

Após serem submetidos a fisioterapia respiratória, limpeza mecânica do esôfago e antibioticoterapia de largo espectro, os doentes selecionados foram operados.

Efetuamos cinco tipos básicos de operações, a saber:

- Cirurgia de Lewis: ${ }^{15}$ Ressecção do esôfago e anastomose esofagogástrica intratorácica manual, acima do arco aórtico, por laparotomia mediana xifoumbilical e toracotomia direita no quinto espaço intercostal, em 31 casos $(51,7 \%)$.

- Cirurgia de Sweet: ${ }^{16}$ Ressecção do esôfago e anastomose esofagogástrica cervical manual, por toracotomia direita no quinto espaço intercostal, laparotomia mediana e cervicotomia lateral esquerda, em 20 casos $(33,3 \%)$.

- Ressecção do esôfago e anastomose esofagocólica manual intratorácica término-lateral em um caso, em função de ressecção gástrica prévia por úlcera duodenal que impedia a mobilização gástrica; e esofagocólica cervical manual término-terminal, em um caso, pela localização do tumor no segmento cervical, próximo ao cricofaríngeo.

- Cirurgias exploradoras, por laparotomia mediana xifoumbilical em um caso e toracotomia direita no quinto espaço intercostal em quatro casos.

- Ostomias, por laparotomia mediana xifoumbilical (gastrostomias), toracotomia direita no quinto espaço intercostal (ressecção do tumor) e cervicotomia lateral esquerda, (esofagostomias) em dois casos.

No seguimento pós-operatório alguns pacientes apresentaram complicações, isoladas ou múltiplas, que agrupamos em: pleuropulmonares, cardíacas, renais, S.A.R.A., sepse, neurológicas, da parede cervical, torácica ou abdominal, deiscência da anastomose, mediastinite, lesão nervosa, lesão linfática, hemorragia, hemorragia digestiva alta e necrose do órgão transposto. Alguns necessitaram reoperações ou procedimentos associados em função das complicações apresentadas. ${ }^{6}$

A correlação entre as variáveis selecionadas, ditas explanatórias, (tempo de história, antecedentes pessoais, hábitos e vícios, perda ponderal, localização do tumor, cirurgia realizada, estádio da doença e caráter da operação), e as complicações pós-operatórias e letalidade hospitalar (variáveis resultantes), obtidas conforme método descrito, foi realizada utilizando-se o programa MULTLR para computador pessoal. Na tentativa de verificar a possível inter-relação entre uma das complicações pós-operatórias e as demais, houve necessidade de considerá-la variável explanatória para efeito de cálculo estatístico.

Como primeira etapa do tratamento estatístico realizamos a análise univariada, verificando o possível efeito de cada variável explanatória sobre as variáveis resultantes.

A análise multivariada foi realizada através do registro das variáveis explanatórias com valor de $\mathrm{p}$ $<0,25^{17}$ utilizando-se o método das inclusões progressivas a partir da variável mais significativa e assim progressivamente, com variáveis explanatórias de coeficientes menos significativos. Nos resultados das análises que envolveram regressão logística múltipla, empregou-se o nível crítico de 5\% ( $\mathrm{p}<0,05)$. 


\section{RESULTADOS}

A localização dos tumores pode ser vista na Tabela 1. O tempo médio de história calculado para os valores registrados foi 170,7 dias (5,7 meses). A maioria dos doentes, $48(80 \%)$, referia ser de até 180 dias o tempo de evolução da doença, com amplitude de zero a 720 dias.

Tabela 1 - Distribuição dos pacientes segundo a localização do tumor.

\begin{tabular}{lcr}
\hline Localização & \multicolumn{2}{c}{ Pacientes } \\
& $\mathbf{N}^{\mathbf{0}}$ & $\mathbf{\%}$ \\
\hline Cervical & 01 & 1,7 \\
Torácico superior & 19 & 31,6 \\
Torácico médio & 27 & 45,0 \\
Torácico inferior & 12 & 20,0 \\
Abdominal & 01 & 1,7 \\
\hline TOTAL & 60 & 100,0 \\
\hline
\end{tabular}

As doenças ou síndromes referidas e confirmadas ao exame físico e exames complementares foram: D.P.O.C. (doença pulmonar obstrutiva crônica), 29 doentes (48,3\%); cardiopatia, nove (15\%); hipertensão arterial, oito (13,3\%); antecedentes de ingestão de cáustico ou corrosivo, dois $(3,3 \%)$ e tuberculose pulmonar, um $(1,7 \%)$. Não relataram doenças ou síndromes pregressas, 19 doentes $(31,7 \%)$. Referiram tabagismo 46 pacientes $(76,7 \%)$; referiram uso de bebida alcoólica 26 pacientes $(43,3 \%)$.

Os registros do peso habitual e do atual nos permitem afirmar que 32 dos pacientes $(53,3 \%)$, haviam perdido até $15 \%$ do peso habitual. Quanto aos 28 restantes: de $15,1 \%$ a $20 \%$, seis pacientes $(10 \%)$; de 20,1 a $25 \%, 15(25 \%)$; e de $25,1 \%$ a $30 \%$, sete doentes $(11,7 \%)$.

Os exames anátomo - patológicos dos produtos de esofagectomia revelaram carcinoma espinocelular em 54 pacientes e adenocarcinoma em um. Nos submetidos a cirurgias exploradoras, não foram colhidas amostras.

Encontravam-se no estádio zero da doença de base dois pacientes $(3,3 \%)$; no estádio 1 , dois (3,3\%); no estádio 2a, 17 (28,3\%); no estádio 2 b, sete $(11,7 \%)$; no estádio $3,23(38,4 \%)$ e no estádio 4 , nove (15\%).

A operação foi caracterizada como: curativa em quatro doentes $(6,7 \%)$ de ressecção macroscópica completa em 27 (45\%), paliativa em 24 (40\%) e exploradora em cinco $(8,3 \%)$.

Apresentaram complicações, isoladas ou múltiplas, 36 pacientes $(60 \%)$ assim distribuídas: pleuropulmonares, 26 (43,3\%); sepse, 10 (16,7\%); deiscência de anastomose cervical, 10/21 (47,6\%); S.A.R.A., sete $(11,7 \%)$; mediastinite, cinco $(8,3 \%)$; cardíacas, quatro $(6,7 \%)$; neurológicas, três $(5 \%)$; deiscência de anastomose intra-torácica, 03/32 (9,4\%); necrose do órgão transposto, 03/53 (5,7\%); da parede cervical, 02/23 (8,7\%); da parede torácica, dois (3,3\%); da parede abdominal, dois $(3,3 \%)$; hemorragia, dois $(3,3 \%)$; insuficiência renal aguda, um $(1,7 \%)$; lesão do nervo laríngeo inferior, um $(1,7 \%)$; lesão do ducto torácico, um $(1,7 \%)$ e hemorragia digestiva alta por úlcera no estômago transposto, um $(1,7 \%$ ) (Tabela 2 ).

Tabela 2 - Distribuição dos pacientes segundo as complicações.

\begin{tabular}{llr}
\hline $\begin{array}{l}\text { Complicações } \\
\text { (natureza) }\end{array}$ & \multicolumn{2}{c}{ Pacientes } \\
$\mathbf{N}^{\mathbf{o}}$ & \% \\
\hline Pleuropulmonares & 26 & 43,3 \\
Cardíacas & 04 & 6,7 \\
Renais & 01 & 1,7 \\
S.A.R.A & 07 & 11,7 \\
Sepse & 10 & 16,7 \\
Neurológicas & 03 & 5,0 \\
Parede cervical (*) & $02 / 23$ & 8,7 \\
Parede torácica & 02 & 3,3 \\
Parede abdominal & 02 & 3,3 \\
Deiscência de anastomose cervical $(*)$ & $10 / 21$ & 47,6 \\
Deiscência de anastomose torácica $(*)$ & $03 / 32$ & 9,4 \\
Mediastinite & 05 & 8,3 \\
Lesão nervosa & 01 & 1,7 \\
Lesão linfática & 01 & 1,7 \\
Hemorragia digestiva alta & 01 & 1,7 \\
Hemorragia & 02 & 3,3 \\
Necrose do órgão transposto $(*)$ & $03 / 53$ & 5,7 \\
Sem complicações & 24 & 40,0 \\
\hline
\end{tabular}

(*) Cálculos realizados em relação ao número total de casos específicos.

Das complicações pleuropulmonares, a broncopneumonia à direita ocorreu em 09/26 pacientes $(34,6 \%)$. A broncopneumonia à direita com hidrotórax ocorreu em $02 / 26$ pacientes $(7,7 \%)$, valores que repetiram-se nos pacientes com bronquite, pneumonia lobar direita, broncopneumonia bilateral e 
broncopneumonia à direita com empiema. $\mathrm{O}$ hemotórax ocorreu em 01/26 doentes (3,8\%), assim como o empiema, a broncopneumonia bilateral com abscesso, a broncopneumonia à esquerda e a broncopneumonia à direita com hidrotórax, empiema e encarceramento pulmonar.

Entre as complicações cardíacas constatamos em dois pacientes a insuficiência cardíaca congestiva e em igual número as arritmias. As complicações neurológicas observadas foram: encefalopatia anóxica, coma hiperosmolar e acidente vascular cerebral isquêmico. $(40 \%)$.

Não apresentaram complicações 24 doentes

Faleceram 16 pacientes $(26,7 \%)$. Analisando as causas conseqüenciais dos óbitos verificamos que em cinco $(31,2 \%)$ foram complicações pleuropulmonares, em quatro $(25 \%)$ à deiscência de anastomose cervical, e em dois $(12,5 \%)$ à necrose do órgão transposto. Em cada um $(6,2 \%)$ dos demais pacientes a encefalopatia anóxica, a miocardiopatia, a deiscência de anastomose intra - torácica, a hemorragia e a lesão intra - torácica do ducto linfático foram as responsáveis pelos óbitos (Tabela 3)

Tabela 3 - Distribuição dos pacientes segundo a causa consequencial do óbito.

\begin{tabular}{lcr}
\hline Causa conseqüencial & \multicolumn{2}{c}{ Pacientes } \\
& $\mathbf{N}^{\mathbf{0}}$ & $\mathbf{\%}$ \\
\hline Pleuropulmonar & 05 & 31,25 \\
Deiscência de anastomose cervical & 04 & 25,00 \\
Deiscência de anastomose torácica & 01 & 6,25 \\
Necrose do órgão transposto & 02 & 12,50 \\
Lesão do ducto torácico & 01 & 6,25 \\
Hemorragia & 01 & 6,25 \\
Arritmia cardíaca & 01 & 6,25 \\
Encefalopatia anóxica & 01 & 6,25 \\
\hline TOTAL & 16 & 100,00 \\
\hline
\end{tabular}

A média global de permanência hospitalar foi de 28,7 dias e a pós-operatória 16,4 dias.

Verificamos, portanto, que em relação às variáveis resultantes, complicações pleuropulmonares, sepse, deiscência de anastomose cervical, mediastinite e óbito, as variáveis explanatórias mais significativas foram, respectivamente: cirurgia paliativa, mediastinite, tumor localizado no segmento torácico superior e sepse. As variáveis explanatórias estudadas, não ti- veram valor significante na análise univariada em relação à resultante deiscência de anastomose torácica.

Constatamos que, em relação à variável resultante complicações pleuropulmonares, as explanatórias associadas mais significativas foram: cirurgia paliativa e S.A.R.A. São interdependentes, permitindo afirmar que nos portadores de câncer do esôfago submetidos a cirurgia paliativa e que desenvolveram S.A.R.A., as complicações pleuropulmonares foram 13,8 vezes mais freqüentes.

Considerando a resultante sepse, as variáveis explanatórias associadas de maior significância foram: mediastinite e S.A.R.A.. Interagem entre si, permitindo afirmar que nos operados de câncer do esôfago, que desenvolveram mediastinite e S.A.R.A., a sepse foi 8,52 vezes mais freqüente.

Analisando a resultante deiscência de anastomose cervical, verificamos que as explanatórias mais significativas foram: tumor de localização no segmento torácico superior, cirurgia paliativa e complicações pleuropulmonares. Atuam de forma independente, permitindo afirmar que nas anastomoses esofagogástricas cervicais a deiscência foi 26 vezes mais freqüente nos doentes operados por tumores localizados no segmento superior, 17 vezes nos submetidos a cirurgia de caráter paliativo e 19 vezes naqueles que desenvolveram complicações pleuropulmonares.

Quanto às resultantes mediastinite e óbito, não houve associação de variáveis explanatórias à sepse, já constatada na análise univariada.

\section{DISCUSSÃO}

As complicações pleuropulmonares no pósoperatório imediato das esofagectomias para câncer de esôfago são freqüentes, podendo alcançar taxas de até $60 \%{ }^{5,7,8,18-21}$. A porcentagem registrada em nossa casuística (43,3\%), é portanto, aceitável.

Entre os fatores de risco que podem exercer alguma influência nas complicações pleuropulmonares são referidos, na literatura: idade avançada, lesão renal, D.P.O.C., PO2, dosagem de albumina e/ou deficiência imunológica - nutricional, tipo de cirurgia, duração de toracotomia, número de incisões, resposta hormonal ao trauma cirúrgico, perda sangüínea operatória e estádio da doença. ${ }^{5,10}$

Algumas das variáveis por nós analisadas, entre as quais a D.P.O.C., a perda ponderal e o estádio do tumor, passíveis de confronto com a literatura 
acima relatada, não demonstraram correlação estatística, tanto em análise uni quanto multivariada, com as complicações pleuropulmonares. Acreditamos que isto tenha ocorrido pelos critérios pré - estabelecidos na seleção dos casos, utilizando provas de função pulmonar e cuidados respiratórios intensivos nos pacientes com D.P.O.C. O mesmo se pode dizer em relação à perda ponderal, com avaliação nutricional pela impressão subjetiva, dosagem de albumina não inferior a $3 \mathrm{mg} \%$ e perda ponderal de no máximo $30 \%$, caracterizando desnutrição.

O estádio da doença, embora não tenha permitido correlação com a ocorrência de complicações pleuropulmonares, contribuiu para maior número de operações paliativas.

Demonstrou-se, pela análise univariada, que a variável explanatória cirurgia paliativa foi a mais significante em relação à resultante complicações pleuropulmonares, estabelecendo-se portanto correlação direta. A associação com S.A.R.A., comprovadamente significante na análise multivariada, em geral se faz em duas situações: hiper - hidratação e infecção.

Considerando de maneira mais específica as complicações pleuropulmonares, verificamos que em 18/26 casos foram de natureza parenquimatosa, sendo a broncopneumonia à direita a mais freqüente (nove casos, 34,6\%). A utilização da toracotomia direita em 59/60 pacientes desta casuística, justificaria estes resultados.

É provável que, com a adoção de programas de rastreamento para diagnóstico precoce, avaliação nutricional criteriosa, utilização rotineira de anestesia peridural, eventualmente acrescida de morfina, uso de cateter de Swan-Ganz respeitando os fundamentos da cinética de água pulmonar extra - vascular, evitando-se intubação pulmonar seletiva e executando modernas técnicas de ventilação mecânica intra operatória, poderemos realizar operações curativas, em maior número, registrando, seguramente, declínio na freqüência das complicações pleuropulmonares e consequentemente da letalidade. ${ }^{18}$

A sepse ocorreu em 10 pacientes $(16,7 \%)$, em cinco desencadeada por complicações pleuropulmonares, em três casos pela deiscência de anastomose cervical e em dois pela necrose do estômago intra-torácico.

Os fatores de risco descritos na literatura que influenciam o desenvolvimento de complicações sépticas fatais são: a idade avançada, o sexo, o estádio da doença e o grau de disfagia. Atuam de modo independente, promovendo desnutrição protéico - calórica e, como consequiência, a sepse. ${ }^{12}$

Os cálculos estatísticos desta casuística ressaltam a mediastinite como a variável explanatória mais significativa correlacionada à sepse na análise univariada e associada à S.A.R.A. em análise multivariada.

A gravidade de uma infecção do mediastino é conhecida, em função das características anatômicas deste compartimento. A presença de tecido areolar frouxo, facilitando a difusão do processo inflamatório e a invasão das bactérias envolvidas, assim como a existência de vasos de grande calibre funcionando como verdadeiras bombas que condicionam disseminação bacteriana, propiciam a sepse. A relação íntima entre infecção e pulmão úmido explica perfeitamente a correlação entre mediastinite e S.A.R.A., obtida pelos cálculos estatísticos.

A mediastinite ocorreu em cinco casos $(8,3 \%)$, todos com evolução letal. É relatada na literatura como responsável por até $20 \%$ dos óbitos, intimamente relacionada às deiscências de anastomose e à utilização da via mediastinal posterior na reconstrução do trânsito. ${ }^{8,19}$

Nos dois casos onde a necrose do estômago transposto determinou a infecção mediastinal, a contaminação direta desencadeando sepse, nos parece ser a seqüência lógica dos fenômenos patológicos. Parece haver nessa sequiência de eventos nítida correlação entre mediastinite, S.A.R.A. e sepse, comprovada estatisticamente.

A análise uni e multivariada em relação à variável resultante mediastinite, constatou correlação direta e isolada à variável explanatória sepse. Assim sendo, verificamos que as correlações estatísticas entre mediastinite e sepse são bilaterais, porém a cronologia de fenômenos patológicos é unidirecional, sentido mediastinite ${ }^{\circledR}$ sepse.

A deiscência da anastomose é referida na literatura como complicação freqüente do câncer do esôfago operado. A cervical é mais frequiente que a torácica, porém de menor gravidade e evolução mais benigna pois é tida como "externa"., 2,6

A associação com mediastinite e/ou sepse, nítida nas torácicas, é também referida nas cervicais, principalmente quando se utiliza a via mediastinal posterior. $8,19,22$

Constatamos pelos cálculos de regressão logística a presença de outras duas variáveis, cirurgia paliativa e complicações pleuropulmonares, atuando 
de maneira independente em relação à resultante deiscência de anastomose cervical.

Para explicar tais resultados podemos afirmar que em nossa casuística, $85 \%$ das cirurgias foram não curativas, e rigorosamente $40 \%$ foram paliativas. Quanto à correlação com as complicações pleuropulmonares, tivemos a impressão de que estas ocorreram isoladamente e na dependência de outros fatores, tais como: tempo de anestesia, número de incisões, tempo operatório e trauma cirúrgico.

Contestamos, mais uma vez, o caráter inócuo e evolução benigna da deiscência de anastomose cervical, pois dos $10 / 21$ pacientes $(47,6 \%)$ em que ocorreu quatro (40\%) faleceram. Destes, três evoluíram com mediastinite e sepse, e dois com complicação pleuropulmonar e sepse, e diabetes descompensada e sepse. Pelo menos nestes casos fica patente a importância secundária das complicações pleuropulmonares como causa conseqüencial de óbito.

Frente a um tumor ressecável localizado no segmento torácico superior, e realizando uma cirurgia de caráter paliativo, a única opção do cirurgião é a anastomose cervical. Para diminuir a frequiência de deiscências (10/21 casos, 47,6\%), considerando as variáveis obtidas nos cálculos de regressão logística, as únicas opções seriam evitar a ocorrência de complicações pleuropulmonares e critérios mais rígidos na indicação de cirurgias paliativas. Embora não haja correlação físiopatológica evidente entre as complicações pleuropulmonares e as deiscências de anastomoses cervicais, a maioria dos cirurgiões sabe que as manifestações pulmonares, em geral, precedem as deiscências na sequiência de fenômenos patológicos.

Entre os que advogam a utilização da esofagectomia sem toracotomia, com o objetivo de diminuir a frequiência de complicações pleuropulmonares e deiscências de anastomose cervical, a maioria não utiliza esta técnica em tumores localizados no segmento superior e médio, pela possibilidade de graves lesões traqueobrônquicas. ${ }^{23} \mathrm{~A}$ utilização das anastomoses mecânicas seria opção a considerar na tentativa de reduzir a frequiência das deiscências. ${ }^{24}$

É provável que, apesar do rigor no critério de seleção dos pacientes, seus perfis constitucional e nutricional não sejam compatíveis com a técnica cirúrgica (Sweet) adotada de rotina nestes casos, justificando, talvez, a frequiência observada de deiscências de anastomose. ${ }^{13,14}$
A freqüência das deiscências de anastomose intra - torácica desta casuística $(03 / 32,9,4 \%)$ é semelhante à registrada na literatura. ${ }^{2,5,25}$

$\mathrm{O}$ fato de que, tanto pela análise univariada quanto pela multivariada, nenhuma das variáveis explanatórias estudadas apresentou valor estatisticamente significante em relação à deiscência de anastomose torácica, parece-nos indicar, frente à casuística selecionada pelos critérios pré - estabelecidos, ser a anastomose esofagogástrica intra-torácica a operação mais adequada, quando factível.

É provável que a cirurgia realizada através de incisões separadas (laparotomia e toracotomia direita), com menores trauma cirúrgico e tempo de anestesia, e com anastomose intra - torácica sem nenhum grau de tensão, passível de envolvimento e proteção com o próprio estômago, sejam fatores responsáveis pelos resultados obtidos. ${ }^{5,19}$

O temor das deiscências de anastomose intra torácica não se confirmou nesta casuística, pois nos três casos constatados, dois evoluíram com fechamento espontâneo. O outro doente faleceu de insuficiência respiratória aguda, após fechamento espontâneo da fístula.

A cirurgia do tipo Lewis, como caráter paliativo ou de ressecção macroscópica completa, quando factível, preenche, nos países onde não se adotam, de rotina, programas de rastreamento para diagnóstico precoce do câncer do esôfago, o principal objetivo do tratamento, ou seja, o alívio da disfagia com restabelecimento da alimentação oral. ${ }^{8,21,23,26}$

Faleceram 16 doentes (26,7\%). Torna-se difícil traçar o paralelo entre a letalidade hospitalar registrada em nossa casuística e da literatura, pela presença de múltiplas variáveis que interferem nos resultados. Entre elas destacaríamos: estádio da doença, idade do paciente, localização do tumor, tipo de cirurgia realizada, associação com radioterapia ou quimioterapia, número de incisões, precocidade do diagnóstico e tamanho da casuística.

As causas conseqüenciais mais freqüentes dos óbitos, segundo a literatura, foram as pleuropulmonares e as deiscências de anastomose associadas ou não à mediastinite e/ou sepse. Estas foram responsáveis por 21 a $41 \%$ dos óbitos.

Verificamos que nossos resultados são similares, pois as complicações pleuropulmonares e as deiscências de anastomose, em igual percentagem, foram responsáveis por $62,5 \%$ dos óbitos. Evidenciase, também, a predominância da deiscência da anastomose cervical $(04 / 16)$ em relação à torácica (01/16) como causadora de $25 \%$ dos óbitos. 
As demais distribuem-se de maneira uniforme, inclusive a deiscência solitária de anastomose intra - torácica, com ligeira predominância para a necrose do órgão transposto $(12,5 \%)$.

Nos dois pacientes em que ocorreu necrose do estômago transposto, não havia deiscência de anastomose, sendo a necrose constatada no fundo gástrico, distante da linha de sutura. ${ }^{8,14,20}$

Dos quatro doentes em que a deiscência da anastomose cervical foi causa conseqüencial dos óbitos, três evoluíram com mediastinite e um sem, significando talvez que esta complicação não seja tão inócua e benigna quanto se apregoa. ${ }^{19}$

Analisando a letalidade hospitalar em relação ao tipo de cirurgia realizada, verificamos que nas anastomoses esofagogástricas intra - torácicas registramos 10/16 óbitos $(32,5 \%)$ e, nas esofagogástricas cervicais $06 / 20(30 \%)$. Os percentuais são muito semelhantes e sem diferença estatística significante. Ainda mais, observando as causas conseqüenciais dos óbitos, constatamos que nas anastomoses esofagogástricas intra - torácicas, os óbitos ocorreram, em sua maioria (40\%), por complicações pleuropulmonares $(04 / 10)$, enquanto nas anastomoses cervicais (04/06), por deiscência (67\%), tendo como denominador comum a sepse.

Os cálculos da análise uni e multivariada, mostrando a sepse como variável explanatória única e independente a correlacionar-se com a resultante óbito, quantifica os resultados obtidos.

Nossos resultados correlacionaram a sepse diretamente aos óbitos constatados e indiretamente à mediastinite, deiscência de anastomose cervical, complicações pleuropulmonares e necrose do estômago transposto. $^{8}$

O declínio da letalidade será possível quando prevenirmos as complicações pleuropulmonares e evitarmos as deiscências de anastomose, graças ao diagnóstico precoce, avaliação nutricional e seleção rigorosa, preparo pulmonar exaustivo, hidratação intra e pós-operatória controladas pelo uso do cateter de Swan-Ganz e obediência irrestrita aos preceitos técnicos de desconexão gástrica e confecção das anastomoses. concluir:

Com base nos resultados obtidos, podemos

- A cirurgia de caráter paliativo que evoluiu com S.A.R.A. influenciou a ocorrência de complicações pleuropulmonares, havendo correlação direta entre a complicação e a concomitância destas variáveis.

- A mediastinite e a S.A.R.A. influenciaram a ocorrência de sepse, havendo correlação direta entre esta complicação e a concomitância das variáveis.

- As cirurgias para tumores localizados no segmento torácico superior, de caráter paliativo, que evoluíram com complicações pleuropulmonares, influenciaram a ocorrência das deiscências das anastomoses cervicais, tendo as variáveis atuado de maneira independente.

- A sepse pós-operatória correlacionou-se de forma direta com os óbitos constatados.

- Não houve correlação entre as variáveis explanatórias estudadas e as deiscências de anastomose intratorácicas.

\begin{abstract}
Objective: The analysis of the variables that can influence on the immediate postoperative complications and hospital mortality of patients with esophageal cancer submitted to surgery. Methods: In a retrospective analysis of data from 60 patients, variables such as provenience, disease history, previous diseases, smoking, alcohol drinking, disease stage, and the nature of the surgery were studied in order to check if they could have played a role on complications and death. Results: The resulting variables: pleuropulmonary complications, sepsis, dehiscence of cervical anastomosis, mediastinitis, and death have been more significantly correlated to the following explanatory variables, respectively: palliative surgery, mediastinitis, tumor located in the superior thoracic segment, and sepsis. By means of univaried analysis, the explanatory variables have showed no significant correlation with dehiscence of thoracic anastomosis. Palliative surgery and respiratory insufficiency were the explanatory variables more significantly associated to the resulting variable pleuropulmonary complications. The interdependence of these variables has allowed to state that pleuropulmonary complications were 13.8 times more frequent in the patients with esophageal cancer submitted to palliative surgery developing respiratory insufficiency. Conclusion: Palliative surgery and tumor located in the superior segment have correlated to pleuropulmonary complications. There was no correlation at all between the variables here studied and dehiscence of intrathoracic anastomosis.
\end{abstract}

Key Words: Esophageal neoplasms; Esophagus; Esofagectomy; Postoperative complications; Hospital mortality. 


\section{REFERÊNCIAS}

1. Ballvé M, Olivé M, Sardá I, et al. - Factores pronósticos de Ia mortalidad postoperatoria del cáncer de esófago. Análisis de 46 casos. Rev Esp Anestesiol Reanim, 1992, 39(1):10-13.

2. Chasseray VM, Kiroff GK, Buard JL, et al. - Cervical or thoracic anastomosis for esophagectomy for carcinoma. Surg Gynecol Obstet, 1989,169(1):55-62.

3. Collard JM, Otte JB, Reynaert M, et al. - Feasibility and effectiveness of en bloc resection of the esophagus for esophageal cancer. Results of a prospective study. Int Surg, 1991, 76(4):209-213.

4. Liu JF, Watson DI, Devitt PG et al. - Risk factor analysis of post-operative mortality in oesophagectomy. Dis Esophagus, 2000, 13(2):130-135.

5. Toyoizumi S, Usui S, Sakamoto A, et al. - Study of risk factors for postoperative pulmonary complications following esophageal cancer surgery : multivariate statistical analysis. Nippon Kyobu Geka Gakkai Zasshi, 1990, 38(2):215-221.

6. Corsi PR, Kanashiro E, Schiola A, et al. - Tratamento cirúrgico das complicações da anastomose esôfago visceral cervical. Rev Col Bras Cir, 2000, 27(5):293-297.

7. Domene CE, Cecconello I, Zilberstein B, et al. - Tunelização esofágica na fístula esofagotraqueobrônquica. Rev Col Bras Cir, 26(2):91-5, 1999.

8. Kimose HH, Lund O, Hasenkam JM, et al. Independent predictors of operative mortality and postoperative complications in surgically treated cacinomas of the oesophagus and cardia - is the aggressive surgical approach worthwhile? Acta Chir Scand, 1990, 156(5):373-382.

9. Peracchia A, Bardini R, Ruol A, et al. Esophagovisceral anastomotic leak. A propective statistical study of predisposing factors. J Thorac Cardiovasc Surg, 1988, 95(4):685-691.

10. Rosa AR, Gurski RR, Schirmer CC, et al. - Survival and prognostic factors in patients with resected epidermoid oesophageal carcinoma. Int Surg, 1999, 84(3):193-198.

11. Saito T, Kuwahara A, Kinoshita T, et al. - Increases in immunoglobulin and complement in patients with esophageal or gastric cancer. Surg Today, 1992, 22(6):537-542.

12. Saito T, Kuwahara A, Shigemitsu Y, et al. - Factors related to malnutrition in patients with esophageal cancer. Nutrition, 1991, 7(2):117-121.

13. Saito T, Shimoda K, Kinoshita T, et al. - Prediction of operative mortality based on impairment of host defense systems in patients with esophageal cancer. J Surg Oncol, 1993, 52(1):1-8.
14. Zhang GH, Fujita H, Yamana H, et al. - Preoperative prediction of mortality following surgery of esophageal cancer. Kurume Med J, 1992, 39(3):159-165.

15. Lewis I - The surgical treatment of carcinoma of the esophagus with especial reference to a new operation for growths of the middle third. Br J Surg, 1946, 34(1):1831.

16. Sweet RH - Late results of surgical treatment of the esophagus. JAMA, 1954, 155:422-425.

17. Hosmer DW, Lameshow S - Applied logistic regression. New York, John Wiley \& Sons, 1989.

18. Heinrichs W, Duda D, Rothmund M, et al. - Changes in pulmonary hemodynamics, gas exchange and extravascular lung fluid in esophageal resection. Anaesthesist, 1988, 37(2):97-104.

19. Wang P, Chien K-Surgical treatment of carcinoma of the esophagus and cardia among the Chinese. Ann Thorac Surg, 1983, 35(2):143-151.

20. Yamanaka H, Hiramatsu Y, Kawaguchi Y, et al. - Surgical treatment for poor-risk patients with carcinoma of the esophagus. Jpn J Surg, 1991, 21(2):178-183.

21. Viana AT - Carcinoma do esôfago : tratamento pela operação de Lewis (resultados imediatos). Rev Col Bras Cir, 1989, 16(6):241-248.

22. Del Grande JC, Hadad CM, Monteiro MS, et al. - Fístula da anastomose esôfago-visceral em doente com câncer do esôfago Rev Col Bras Cir, 1993, 20(6):310-315.

23. Fok M, Siu K, Wong J - A comparison of transhiatal and transthoracic resection for carcinoma of the thoracic esophagus. Am J Surg, 1989, 158(5):414-419.

24. McManus KG, Ritchie AJ, McGuigan J, et al. - Sutures, staplers, leaks and strictures. A review of anastomoses in oesophageal resection at Royal Victoria Hospital, Belfast 1977-1986. Eur J Cardiothorac Surg, 1990, 4(2):97-100.

25. Sons HU, Borchard F-Cancer of the distal esophagus and cardia. Incidence, tumorous, infiltration, and metastatic spread. Ann Surg, 1986, 203(2):188-195.

26. Corsi PR, Viana AT, Gagliardi D, et al. - A gastrostomia no tratamento paliativo do câncer do esôfago. Rev Col Bras Cir, 1994, 21(3):105-111.

Endereço para correspondência:

Paulo Roberto Corsi

Av. Brigadeiro Faria Lima, ${ }^{\circ} 1571-2^{\circ}$ andar

Pinheiros

01452-001 - São Paulo - SP 